\title{
Oscillations of Solutions of Neutral Nonlinear Differential Equations
}

\author{
Jinyu Wang*, Min Xi, Ailing Xiao \\ School of Finance, Guangdong University of Foreign Studies, Guangzhou, China \\ Email address: \\ jinyuw126@126.com (Jinyu Wang), sheamee@hotmail.com (Min Xi), alxiao181@126.com (Ailing Xiao) \\ *Corresponding author
}

To cite this article:

Jinyu Wang, Min Xi, Ailing Xiao. Oscillations of Solutions of Neutral Nonlinear Differential Equations. Applied and Computational Mathematics. Vol. 7, No. 3, 2018, pp. 112-120. doi: 10.11648/j.acm.20180703.16

Received: May 26, 2018; Accepted: July 3, 2018; Published: July 19, 2018

\begin{abstract}
This paper aims to establish a new class of differential equations and study the oscillatory behavior of a kind of first-order neutral nonlinear differential equation with time delay arguments. The oscillatory properties of the solutions of the type of first order neutral functional differential equations applied in chemomedical problems are studied. Sufficient conditions for the oscillations of solutions of the above equations are obtained. Also, some results which demonstrate in literature [1-4] will be extended, and the paper focuses on expanding the main finding of literature [2,3]. Moreover, a new kind of method to be used to discuss the properties of oscillation of the first-order neutral nonlinear differential equations and some theorems are obtained in the paper.
\end{abstract}

Keywords: Oscillation, Differential Equations, Neutral, Piecewise Constant Arguments

\section{Introduction}

The oscillation theory of differential equations with deviating arguments is a relatively new and rapidly developing branch of the theory of ordinary differential equation, numerous research papers have been devoted to this study. Recently, attempts have been made by many mathematicians to develop the oscillation theory of differential equations with deviating arguments. The mathematical modeling of several real-world problems leads to differential equations rely more on the past history rather than on the present. The models may have discrete time delays as well as distributed lags or delays originated in [5]. Bernoulli (1728) studied the problem of sound vibrating in a tube of finite length and investigated the properties of first order Ordinary Differential Equation With Deviating Arguments (ODEWDA). Miskis investigated several oscillation problems of first order ODEWDA, which are recorded in his book. Since 1950 oscillation theory of ODEWDA has received the attention of several applied mathematicians as well as other scientists around the world. There are two main reasons to pursue this research, firstly, theoretically it is well-known that the ODE $y^{\prime}(t)+p(t) y(t)=0 \quad p(t) \in C\left(R^{+}\right)$has no the oscillatory solution, but the equation $y^{\prime}(t)+y\left(t-\frac{\pi}{2}\right)=0$ has the oscillatory solution $y=\sin t$. Therefore, the oscillation of this type of first order equation is caused by deviating arguments. Secondly, this problem arises in many industrial and scientific problems, for example the literature of [6]. The first systematic research of the oscillation of first order equations with deviating arguments was given in the book "Linear differential equations with retarded argument" by Miskis, A. D.

The new development of the oscillation theory of differential equation with deviating arguments has two cases, those are the research of oscillatory solution of partial differential equation with deviating arguments and the research of oscillatory solution of difference equation with deviating arguments. The difference equation has been considered in its own right as a method of biology model of single species with nonoverlapping generation, on the other hand, some methods for the latter in the special case when the deviation of argument vanishes at individual points have been used to investigate differential equations with piecewise constant delays, this note continues the investigation of differential equations with piecewise constant arguments (EPCA) originated in literature [7]. They are closely related to 
impulse and loaded equation, especially, to difference equation of a discrete argument, these equations have the structure of continuous dynamical systems within intervals of certain length. Continuity of a solution at a point joining any two consecutive intervals implies recursion for the solution at such points, and the equations are thus similar in structure to those found in certain "sequential-continuous" models of disease dynamics. The cited works show that all types of EPCA share similar characteristics. First of all, it is natural pose the initial value problem for such equations not on interval but at a number of individual points, second, two-sided solutions exist for all types of EPCA, finally, since EPCA combine the features of both differential and difference equations, their asymptotic-behavior as tending to infinity resembles in some cases the solutions growth of differential equation, while in other it inherits the properties of difference equation.

In recent years there has been a growing interesting in oscillation theory of the retarded and advanced functional differential equations with piecewise constant arguments, see, for example in literature [6]-[16]. And there were some papers on oscillatory properties of neutral functional differential equations with piecewise constant arguments [17]-[32], especially, there has been a lot of activities concerning the oscillatory behavior of a kind of first-order neutral linear differential equations. But there is hardly any work at the time concerning the oscillatory behavior of a kind of first-order neutral nonlinear differential equations with time delay arguments.

\section{Preliminaries}

In this paper, the first-order neutral nonlinear differential inequalities with time delay arguments and the neutral nonlinear functional differential equation with time delay arguments are considered

$$
\begin{gathered}
{\left[x(t)-\sum_{j=1}^{n} c_{j} x\left(t-\theta_{j}\right)\right]^{\prime}+a(t) g\left[x\left(t-\sigma_{1}(t)\right), x\left(t-\sigma_{2}(t)\right), \ldots, x\left(t-\sigma_{k}(t)\right)\right]} \\
\quad+p(t) f\left[x\left(t-\tau_{1}(t)\right), x\left(t-\tau_{2}(t)\right), \ldots, x\left(t-\tau_{k}(t)\right)\right] \leq 0, \\
{\left[x(t)-\sum_{j=1}^{n} c_{j} x\left(t-\theta_{j}\right)\right]^{\prime}+a(t) g\left[x\left(t-\sigma_{1}(t)\right), x\left(t-\sigma_{2}(t)\right), \ldots, x\left(t-\sigma_{k}(t)\right)\right]} \\
\quad+p(t) f\left[x\left(t-\tau_{1}(t)\right), x\left(t-\tau_{2}(t)\right), \ldots, x\left(t-\tau_{k}(t)\right)\right] \geq 0
\end{gathered}
$$

and

$$
\begin{gathered}
{\left[x(t)-\sum_{j=1}^{n} c_{j} x\left(t-\theta_{j}\right)\right]^{\prime}+a(t) g\left[x\left(t-\sigma_{1}(t)\right), x\left(t-\sigma_{2}(t)\right), \ldots, x\left(t-\sigma_{k}(t)\right)\right]} \\
+p(t) f\left[x\left(t-\tau_{1}(t)\right), x\left(t-\tau_{2}(t)\right), \ldots, x\left(t-\tau_{k}(t)\right)\right]=0,
\end{gathered}
$$

where $\mathrm{a}(\mathrm{t}), \mathrm{p}(\mathrm{t}), \sigma_{\mathrm{i}}(\mathrm{t})$ and $\tau_{\mathrm{i}}(\mathrm{t})$ are continuous functions for $t \in R^{+}, \mathrm{i}=1,2, \ldots, \mathrm{k}$. such that $\mathrm{a}(\mathrm{t}) \geq 0, \quad \mathrm{p}(\mathrm{t})>0, \quad 0 \leq \sigma_{\mathrm{i}}(\mathrm{t}) \leq \mathrm{M}$ and $0<\mathrm{m} \leq \tau_{\mathrm{i}}(\mathrm{t}) \leq \mathrm{M}\left(\mathrm{m}, \mathrm{M}\right.$ are positive constants). $\mathrm{c}_{\mathrm{j}}, \theta_{\mathrm{j}}$ are positive constants too, $0 \leq \sum_{\mathrm{j}=1}^{\mathrm{n}} \mathrm{c}_{\mathrm{j}}<1,0<\theta_{1}<\theta_{2}<\cdots<\theta_{\mathrm{n}}$. Also suppose that the functions $\mathrm{f}$ and $\mathrm{g}$ satisfy the following condition:

(A) $\mathrm{f}$ ( or $\mathrm{g}$ ) is continuous on $\mathrm{R}^{\mathrm{k}}$ and such that

$$
\mathrm{y}_{\mathrm{j}}>0 \text {, for } \mathrm{j}=1,2, \ldots, \mathrm{k} . \Rightarrow \mathrm{f}\left(\mathrm{y}_{1}, \mathrm{y}_{2}, \cdots, \mathrm{y}_{\mathrm{k}}\right)>0,\left(\text { or } \mathrm{g}\left(\mathrm{y}_{1}, \mathrm{y}_{2}, \cdots, \mathrm{y}_{\mathrm{k}}\right)>0\right)
$$

and

$$
\mathrm{y}_{\mathrm{j}}<0 \text {, for } \mathrm{j}=1,2, \ldots, \mathrm{k} . \Rightarrow \mathrm{f}\left(\mathrm{y}_{1}, \mathrm{y}_{2}, \cdots, \mathrm{y}_{\mathrm{k}}\right)<0,\left(\text { or } \mathrm{g}\left(\mathrm{y}_{1}, \mathrm{y}_{2}, \cdots, \mathrm{y}_{\mathrm{k}}\right)<0\right) \text {. }
$$

Some sufficient conditions are given as follows:

1. Differential inequality (1) has no eventually positive solutions;

2. Differential inequality (2) has no eventually negative solutions;
3. Differential equation (3) has only oscillatory solutions.

As it is customary, a solution is said to be oscillatory if it has arbitrarily large zeros. 


\section{Two Lemmas}

Lemma 1 Suppose that $\mathrm{x}(\mathrm{t})$ is an eventually positive solution of (1). Let

$$
z(t)=x(t)-\sum_{j=1}^{n} c_{j} x\left(t-\theta_{j}\right)
$$

Then there is a $T \geq t_{0}$, such that $z^{\prime}(t)<0$, $z(t)>0$ for $t \geq T$.

Proof Suppose that $x(t)>0, x\left(t-\theta_{n}-M\right)>0$, for $t \geq T \geq t_{0}$. From (1), we get that $z^{\prime}(t)<0$, so $z(t)$ is strictly decreasing for $t>T$.

Let $1 \operatorname{im}_{\mathrm{t} \rightarrow \infty} \mathrm{z}(\mathrm{t})=\mathrm{L}$, then $\mathrm{L}$ is a finite constant or $-\infty$.

It can be proved that $L=-\infty$ is impossible. Assume that $L=-\infty$, then we get the fact $x(t)$ is unbounded from (4). So there is a sequence $\left\{t_{k}\right\}, t_{k} \rightarrow \infty(k \rightarrow \infty)$ such that

$$
\begin{gathered}
x\left(t_{k}\right)=\max _{s \leq t_{k}}\{x(s)\}, \underset{t \rightarrow \infty}{1 \operatorname{man}_{x} x\left(t_{k}\right)=+\infty} \\
z\left(t_{k}\right)=x\left(t_{k}\right)-\sum_{j=1}^{n} c_{j} x\left(t_{k}-\theta_{j}\right) \geq x\left(t_{k}\right)-\sum_{j=1}^{n} c_{j} x\left(t_{k}\right) \\
=\left(1-\sum_{j=1}^{n} c_{j}\right) x\left(t_{k}\right) \rightarrow+\infty,(k \rightarrow \infty)\left(0 \leq \sum_{j=1}^{n} c_{j}<1\right)
\end{gathered}
$$

This is a contradiction to $\mathrm{L}=-\infty$. Hence, $\mathrm{L}$ is a finite constant. Thus, $z(t)$ is bounded and $x(t)$ is bounded too. Then $\overline{1} \operatorname{im}_{\mathrm{t} \rightarrow \infty}^{\mathrm{x}}(\mathrm{t})<\infty$.

It is easy to prove that $\mathrm{L}<0$ is impossible from (5). Now we shall prove that $\mathrm{L}>0$.

Take $\left\{t_{k}\right\}$ such that $t_{k} \rightarrow \infty(k \rightarrow \infty)$ and

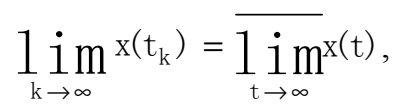

Then

$$
\begin{aligned}
& L=1 \lim _{k \rightarrow \infty} z\left(t_{k}\right)=1 \operatorname{im}_{k \rightarrow \infty}\left[x\left(t_{k}\right)-\sum_{j=1}^{n} c_{j} x\left(t_{k}-\theta_{j}\right)\right] \\
& =\lim _{\mathrm{k} \rightarrow \infty} \mathrm{x}\left(\mathrm{t}_{\mathrm{k}}\right)-1 \operatorname{im}_{\mathrm{k} \rightarrow \infty} \sum_{j=1}^{\mathrm{n}} \mathrm{c}_{\mathrm{j}} \mathrm{x}\left(\mathrm{t}_{\mathrm{k}}-\theta_{\mathrm{j}}\right) .
\end{aligned}
$$

Since $\left\{x\left(t_{k}-\theta_{j}\right)\right\}(j=1,2, \ldots, n$. $)$ is bounded, we have a subsequence $\left\{t_{k_{i}}\right\} \subset\left\{t_{k}\right\}$ such that $1 \operatorname{im}_{\mathrm{i} \rightarrow \infty} x\left(t_{k_{i}}-\theta_{j}\right)(j=1,2, \ldots$, n. $)$ exist.

So

$$
\begin{aligned}
& L=1 \operatorname{im}_{i \rightarrow \infty} z\left(t_{k_{i}}\right)=1 \operatorname{im}_{k \rightarrow \infty}\left[x\left(t_{k_{i}}\right)-\sum_{j=1}^{n} c_{j} x\left(t_{k_{i}}-\theta_{j}\right)\right] \\
& =1 \operatorname{im}_{i \rightarrow \infty} x\left(t_{k_{i}}\right)-1 \operatorname{im}_{i \rightarrow \infty} \sum_{j=1}^{n} c_{j} x\left(t_{k_{i}}-\theta_{j}\right) \\
& \geq \overline{\lim _{t \rightarrow \infty}} x(t)-\sum_{j=1}^{n} c_{j} \overline{\lim _{t \rightarrow \infty}} x(t)
\end{aligned}
$$

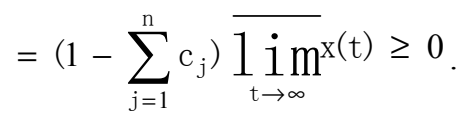

Since $z(t)$ is strictly decreasing, so $z(t)>0$ for $t \geq T$. we obtain that

$$
z^{\prime}(t)<0, z(t)>0 \text { for } t \geq T .
$$

Lemma 2 Suppose that $\mathrm{x}(\mathrm{t})$ is an eventually positive solution of (1). Let

$$
z(t)=x(t)-\sum_{j=1}^{n} c_{j} x\left(t-\theta_{j}\right)
$$

and

$$
\omega(t)=\frac{z(t-m)}{z(t)}
$$

If $\underset{\mathrm{t} \rightarrow \infty}{\operatorname{im}} \inf \omega(\mathrm{t})=\mathrm{X} \geq 1$, and $X$ is finite then $\lim _{\mathrm{t} \rightarrow \infty} \mathrm{x}(\mathrm{t})=0$.

Proof By Lemma 1, there is a $T \geq t_{0}$, such that $z^{\prime}(t)<0, z(t)>0$ for $t \geq T$, then

$$
\lim _{\mathrm{t} \rightarrow \infty} \mathrm{z}(\mathrm{t})=\mathrm{L},(0 \leq \mathrm{L}<+\infty)
$$

Integrating both sides of (1) from $t-m$ to $t$, for $t>t_{0}+3 M$

$$
z(t)-z(t-m)+\int_{t-m}^{t} a(s) g\left[x\left(s-\sigma_{1}(s)\right), \cdots, x\left(s-\sigma_{k}(s)\right)\right] d s
$$




$$
+\int_{t-m}^{t} p(s) f\left[x\left(s-\tau_{1}(s), \cdots, x\left(s-\tau_{k}(s)\right)\right] d s \leq 0, t>t_{0}+3 M .\right.
$$

If $0<\mathrm{L}<+\infty$, which contradicts the above inequality.

Therefore $\lim _{\mathrm{t} \rightarrow \infty} \mathrm{z}(\mathrm{t})=0$, and $\lim _{\mathrm{t} \rightarrow \infty} \mathrm{x}(\mathrm{t})=0$.

$$
=\left(1-\sum_{j=1}^{n} c_{j}\right) \overline{\lim _{t \rightarrow \infty}} x(t) \neq 0,
$$

Therefore $\boldsymbol{z}(\boldsymbol{t})$ is bounded and so is $\boldsymbol{x}(\boldsymbol{t})$.

If $\lim _{\mathrm{t} \rightarrow \infty} \mathrm{x}(\mathrm{t}) \neq 0$, Assume that $0<\overline{\lim _{\mathrm{t} \rightarrow \infty} \mathrm{x}(\mathrm{t})}<\infty$. Take $\left\{t_{k}\right\}$ such that $t_{k} \rightarrow \infty(k \rightarrow \infty)$, the proof is similar $\quad \lim _{t \rightarrow \infty} x(t)=0$.

to the proof of (5) in Lemma 1.

It can get

$$
\begin{aligned}
& \lim _{k \rightarrow \infty} x\left(t_{k}\right)=\overline{\lim _{t \rightarrow \infty}} x(t) \\
& L \geq \overline{\lim _{t \rightarrow \infty}} x(t)-\sum_{j=1}^{n} c_{j} \overline{\lim _{t \rightarrow \infty}}(t) \\
& {\left[x(t)-\sum_{j=1}^{n} c_{j} x\left(t-\theta_{j}\right)\right]^{\prime}+a(t) g\left[x\left(t-\sigma_{1}(t)\right), x\left(t-\sigma_{2}(t)\right), \ldots, x\left(t-\sigma_{k}(t)\right)\right]} \\
& +p(t) f\left[x\left(t-\tau_{1}(t)\right), x\left(t-\tau_{2}(t)\right), \ldots, x\left(t-\tau_{k}(t)\right)\right] \leq 0,
\end{aligned}
$$

\section{Oscillations of the Neutral Nonlinear}

Theorem 1 Consider the delay differential inequality where $\mathrm{a}(\mathrm{t}), \mathrm{p}(\mathrm{t}), \sigma_{\mathrm{i}}(\mathrm{t})$ and $\tau_{\mathrm{i}}(\mathrm{t})$ are continuous functions for $\quad t \in R^{+}, \quad i=1,2, \ldots, k$. such that $\mathrm{a}(\mathrm{t}) \geq 0, \quad \mathrm{p}(\mathrm{t})>0, \quad 0 \leq \sigma_{\mathrm{i}}(\mathrm{t}) \leq \mathrm{M} \quad$ and

$0<\mathrm{m} \leq \tau_{\mathrm{i}}(\mathrm{t}) \leq \mathrm{M}$ ( $\mathrm{m}, \mathrm{M}$ are positive constants). $\mathrm{c}_{\mathrm{j}}, \theta_{\mathrm{j}}$ are positive constants too, $0 \leq \sum_{j=1}^{\mathrm{n}} \mathrm{c}_{\mathrm{j}}<1$, $0<\theta_{1}<\theta_{2}<\cdots<\theta_{\mathrm{n}} \leq \mathrm{M}$. Also suppose that the functions $f$ and $g$ satisfy the condition (A). Assume that there exist nonnegative numbers $\alpha_{i}, i=1,2, \ldots, k$, with $\sum_{i=1}^{k} \alpha_{i}=1$ and $\sum_{i=1}^{k} \beta_{i}=1$ such that

$$
\left.\frac{1}{H} \lim _{t \rightarrow \infty}{ }^{i n f} \int_{t-m}^{t} p(s) d s>\frac{1}{e} \exp \left(-\frac{1}{G}\right] \operatorname{imm}_{t \rightarrow \infty} \inf ^{t} \int_{t-m} a(s) d s\right)
$$

and

$$
\lim _{t \rightarrow \infty} \inf \int_{t-\frac{m}{2}}^{t} \frac{1}{H} p(s) d s>0
$$

Then (1) has no eventually positive solutions.

Proof The existence of an eventually positive solution will be proved that it leads to a contradiction. To this end suppose that $x(t)$ is solution of (1) such that for $t_{0}$ sufficiently large

$$
\mathrm{x}(\mathrm{t})>0, \forall \mathrm{t}>\mathrm{t}_{0}
$$

They can choose a $t_{1}>t_{0}+M$ such that $x\left(t-\sigma_{i}(t)\right)>0, x\left(t-\tau_{i}(t)\right)>0, \quad i=1,2, \ldots, k$. for $t>t_{1}$. let $z(t)=x(t)-\sum_{j=1}^{n} c_{j} x\left(t-\theta_{j}\right)$, and observing that the functions $\mathrm{f}$ and $\mathrm{g}$ satisfy the condition (A), and thus from (1) and Lemma1, you obtain $z^{\prime}(t)<0, z(t)>0$ for $t>t_{0}+M$, i.e. $z(t)$ is strictly decreasing for $t>t_{0}+M$, Hence, you have $z(t)<z(t-m)$ and $z(t)<x(t)$ for $t>t_{0}+2 M$ 
Set

$$
\begin{gathered}
\omega(\mathrm{t})=\frac{\mathrm{z}(\mathrm{t}-\mathrm{m})}{\mathrm{z}(\mathrm{t})}, \forall \mathrm{t}>\mathrm{t}_{0}+2 \mathrm{M} \quad \text { (8) they obtain } \\
\quad \frac{\mathrm{z}^{\prime}(\mathrm{t})}{\mathrm{z}(\mathrm{t})}+\mathrm{a}(\mathrm{t}) \frac{\mathrm{g}\left[\mathrm{x}\left(\mathrm{t}-\sigma_{1}(\mathrm{t})\right), \cdots, \mathrm{x}\left(\mathrm{t}-\sigma_{\mathrm{k}}(\mathrm{t})\right)\right]}{\mathrm{z}(\mathrm{t})} \\
+\mathrm{p}(\mathrm{t}) \frac{\mathrm{g}\left[\mathrm{x}\left(\mathrm{t}-\tau_{1}(\mathrm{t})\right), \cdots, \mathrm{x}\left(\mathrm{t}-\tau_{\mathrm{k}}(\mathrm{t})\right)\right]}{\mathrm{z}(\mathrm{t})} \leq 0, \forall \mathrm{t}>\mathrm{t}_{0}+2 \mathrm{M}
\end{gathered}
$$

Then $\omega(t)>1$ and dividing both sides of (1) by $z(t)$,

Integrating both sides of (9) from $t-m$ to $t$, for $t>t_{0}+3 M$, we have

$$
\begin{aligned}
& \ln z(t)-\ln z(t-m)+\int_{t-m}^{t} a(s) \frac{g\left[x\left(s-\sigma_{1}(s)\right), \cdots, x\left(s-\sigma_{k}(s)\right)\right]}{z(s)} d s \\
& +\int_{t-m}^{t} p(s) \frac{g\left[x\left(s-\tau_{1}(s)\right), \cdots, x\left(s-\tau_{k}(s)\right)\right]}{z(s)} d s \leq 0, \forall t>t_{0}+3 M
\end{aligned}
$$

From which and (8), we obtain

$$
\begin{aligned}
& \ln \omega(t) \geq \int_{t-m}^{t} a(s) \frac{g\left[x\left(s-\sigma_{1}(s)\right), \cdots, x\left(s-\sigma_{k}(s)\right)\right]}{\prod_{i=1}^{k} x^{\alpha_{i}}\left(s-\sigma_{i}(s)\right)} \frac{\prod_{i=1}^{k} x^{\alpha_{i}}\left(s-\sigma_{i}(s)\right)}{z(s)} d s \\
& +\int_{t-m}^{t} p(s) \frac{f\left[x\left(s-\tau_{1}(s)\right), \cdots, x\left(s-\tau_{k}(s)\right)\right]}{\prod_{i=1}^{k} x^{\beta_{i}}\left(s-\sigma_{i}(s)\right)} \frac{\prod_{i=1}^{k} x^{\beta_{i}}\left(s-\tau_{i}(s)\right)}{z(s)} d s \\
& \forall \mathrm{t}>\mathrm{t}_{0}+3 \mathrm{M}
\end{aligned}
$$

Notice that $z(t)<x(t)$ for $t>t_{0}+2 M$.

$$
\begin{aligned}
& \text { And } \ln \omega(\mathrm{t}) \geq \inf _{\mathrm{s}>\mathrm{t}-\mathrm{m}} \frac{\mathrm{g}\left[\mathrm{x}\left(\mathrm{s}-\sigma_{1}(\mathrm{~s})\right), \cdots, \mathrm{x}\left(\mathrm{s}-\sigma_{\mathrm{k}}(\mathrm{s})\right)\right]}{\prod_{i=1}^{\mathrm{k}} \mathrm{x}^{\alpha_{i}}\left(\mathrm{~s}-\sigma_{\mathrm{i}}(\mathrm{s})\right)} \frac{\prod_{\mathrm{i}=1}^{\mathrm{k}} \mathrm{z}^{\alpha_{\mathrm{i}}}\left(\mathrm{s}-\sigma_{\mathrm{i}}(\mathrm{s})\right)}{\mathrm{z}(\mathrm{s})} \int_{\mathrm{t}-\mathrm{m}}^{\mathrm{t}} \mathrm{a}(\mathrm{s}) \mathrm{ds} \\
& +\int_{t-m}^{t} p(s) \inf _{s>t-m} \frac{f\left[x\left(s-\tau_{1}(s)\right), \cdots, x\left(s-\tau_{k}(s)\right)\right]}{\prod_{i=1}^{k} x^{\beta_{i}}\left(s-\sigma_{i}(s)\right)} \frac{\prod_{i=1}^{k} z^{\beta_{i}}\left(s-\tau_{i}(s)\right)}{z(s)} d s
\end{aligned}
$$

$$
\forall \mathrm{t}>\mathrm{t}_{0}+3 \mathrm{M}
$$

$$
\frac{\prod_{i=1}^{k} x^{\alpha_{i}}\left(s-\sigma_{i}(s)\right)}{z(s)} \geq \frac{\prod_{i=1}^{k} z^{\alpha_{i}}\left(s-\sigma_{i}(s)\right)}{z(s)} \geq 1
$$$$
\frac{\prod_{i=1}^{k} z^{\beta_{i}}\left(s-\tau_{i}(s)\right)}{z(s)} \geq \frac{\prod_{i=1}^{k} z^{\beta_{i}}(s-m)}{z(s)} \geq \frac{z(s-m)}{z(s)}=\omega(s)
$$ 


$$
+\inf _{0<y_{i}<x(t-m-M)} \frac{f\left(y_{1}, y_{2}, \cdots, y_{k}\right)}{\prod_{i=1}^{k} y_{i}^{\beta_{i}}} \int_{t-m}^{t} p(s) \omega(s) d s \quad \forall t>t_{0}+3 M
$$

Integrating both sides of (1) from $t-\frac{m}{2}$ to $t$, for $t>t_{0}+\frac{5}{2} M$ and observe that $z(t)$ is strictly decreasing, it can be achieved that

$$
\begin{gathered}
\frac{z(t)}{z(s)}<1 \text {, and } \frac{z(t-m)}{z\left(s-\tau_{i}(s)\right)}<1 \text { for } t-\frac{m}{2}<s<t, s-\tau_{i}(s)<s-m<t-m \\
z(t)-z\left(t-\frac{m}{2}\right)+z(t) \inf _{s>t-\frac{m}{2}} \frac{g\left[x\left(s-\sigma_{1}(s)\right), \cdots, x\left(s-\sigma_{k}(s)\right)\right]}{\prod_{i=1}^{k} x^{\alpha_{i}}\left(s-\sigma_{i}(s)\right)} \int_{t-\frac{m}{2}}^{t} a(s) d s \\
+z(t-m) \inf _{s>t-\frac{m}{2}} \frac{f\left[x\left(s-\tau_{1}(s)\right), \cdots, x\left(s-\tau_{k}(s)\right)\right]}{\prod_{i=1}^{k} x^{\beta_{i}}\left(s-\sigma_{i}(s)\right)} \int_{t-\frac{m}{2}}^{t} p(s) d s \leq 0
\end{gathered}
$$

Dividing the last inequality first by $z(t)$ and then by $z\left(t-\frac{m}{2}\right)$, we obtain, respectively:

$$
\begin{gathered}
1-\frac{z\left(t-\frac{m}{2}\right)}{z(t)}+\inf _{s>t-\frac{m}{2}} \frac{g\left[x\left(s-\sigma_{1}(s)\right), \cdots, x\left(s-\sigma_{k}(s)\right)\right]}{\prod_{i=1}^{k} x^{\alpha_{i}}\left(s-\sigma_{i}(s)\right)} \int_{t-\frac{m}{2}}^{t} a(s) d s \\
+\frac{z(t-m)}{z(t)} \inf _{s>t-\frac{m}{2}} \frac{f\left[x\left(s-\tau_{1}(s)\right), \cdots, x\left(s-\tau_{k}(s)\right)\right]}{\prod_{i=1}^{k} x^{\beta_{i}}\left(s-\sigma_{i}(s)\right)} \int_{t-\frac{m}{2}}^{t} p(s) d s \leq 0 \quad \forall t>t_{0}+\frac{5}{2} M
\end{gathered}
$$

and

$$
\begin{gathered}
\frac{z(t)}{z\left(t-\frac{m}{2}\right)}-1+\frac{z(t)}{z\left(t-\frac{m}{2}\right)} \inf _{s>t-\frac{m}{2}} \frac{g\left[x\left(s-\sigma_{1}(s)\right), \cdots, x\left(s-\sigma_{k}(s)\right)\right]}{\prod_{i=1}^{k} x^{\alpha_{i}}\left(s-\sigma_{i}(s)\right)} \int_{t-\frac{m}{2}}^{t} a(s) d s \\
+\frac{z(t-m)}{z\left(t-\frac{m}{2}\right)} \inf _{s>t-\frac{m}{2}} \frac{f\left[x\left(s-\tau_{1}(s)\right), \cdots, x\left(s-\tau_{k}(s)\right)\right]}{\prod_{i=1}^{k} x^{\beta_{i}}\left(s-\sigma_{i}(s)\right)} \int_{t-\frac{m}{2}}^{t} p(s) d s \leq 0 \quad \forall t>t_{0}+\frac{5}{2} M
\end{gathered}
$$

Let $\lim _{\mathrm{t} \rightarrow \infty} \inf \omega(\mathrm{t})=\mathrm{X}$

Then we have $X \geq 1$. Now, the following two possible cases are considered:

Case $1 X$ is finite. We have $z^{\prime}(t)<0$, for $t>t_{0}+M$ and therefore from (1),

We find $\lim _{\mathrm{t} \rightarrow \infty} \mathrm{z}(\mathrm{t})=0$

Notice that $z(t)=x(t)-\sum_{j=1}^{n} c_{j} x\left(t-\theta_{j}\right)$ 
By Lemma 2, so $1 \operatorname{im}_{\mathrm{t} \rightarrow \infty} \mathrm{x}(\mathrm{t})=0$

Taking limit inferiors on both sides of (8), we obtain

$$
\ln X \geq \frac{1}{G} 1 \operatorname{im}_{t \rightarrow \infty} \inf \int_{t-m}^{t} a(s) d s+\frac{X}{H} 1 \operatorname{im}_{t \rightarrow \infty} \text { inf } \int_{t-m}^{t} p(s) d s
$$

Using the fact that $\ln X-a X \leq-\ln a-1$ for $X \geq 1$, so we get

$$
\left.\ln X-\frac{X}{H} 1 \operatorname{im}_{t \rightarrow \infty} \inf \int_{t-m}^{t} a(s) d s \leq-\ln \left(\frac{1}{H}\right] \operatorname{im}_{t \rightarrow \infty} \inf \int_{t-m}^{t} p(s) d s\right)-1
$$

and

$$
\frac{1}{\mathrm{H}} \underset{\mathrm{t} \rightarrow \infty}{\inf } \int_{t-m}^{t} p(s) d s \leq \frac{1}{\mathrm{e}} \exp \left(-\frac{1}{\mathrm{G}} \underset{\mathrm{t} \rightarrow \infty}{\operatorname{im}} \mathrm{inf}_{\mathrm{t}-\mathrm{m}}^{\mathrm{t}} \mathrm{a}(\mathrm{s}) \mathrm{ds}\right)
$$

The last inequality contradicts hypothesis (4).

Case $2 \mathrm{X}$ is infinite. That is

$$
\lim _{\mathrm{t} \rightarrow \infty} \frac{\mathrm{z}(\mathrm{t}-\mathrm{m})}{\mathrm{z}(\mathrm{t})}=+\infty
$$

In view of (5) and the fact that $a(t) \geq 0$, from (9), we get

$$
\lim _{\mathrm{t} \rightarrow \infty} \frac{\mathrm{z}\left(\mathrm{t}-\frac{\mathrm{m}}{2}\right)}{\mathrm{z}(\mathrm{t})}=+\infty \text {. }
$$

$$
\lim _{t \rightarrow \infty} \frac{z(t-m)}{z\left(t-\frac{m}{2}\right)}=+\infty
$$

which contradicts (12). So (1) has no eventually positive solutions.

Theorem 2 Consider the delay differential inequality.

And, therefore

$$
\begin{gathered}
{\left[x(t)-\sum_{j=1}^{n} c_{j} x\left(t-\theta_{j}\right)\right]^{\prime}+a(t) g\left[x\left(t-\sigma_{1}(t)\right), x\left(t-\sigma_{2}(t)\right), \ldots, x\left(t-\sigma_{k}(t)\right)\right]} \\
\quad+p(t) f\left[x\left(t-\tau_{1}(t)\right), x\left(t-\tau_{2}(t)\right), \ldots, x\left(t-\tau_{k}(t)\right)\right] \geq 0,
\end{gathered}
$$

where $a(t), p(t), \sigma_{i}(t) \tau_{i}(t) c_{j}, \theta_{j}, f$ and $g$ satisfy the condition of Theorem 1 ,

$i=1,2, \ldots, k . j=1,2, \ldots, n$. Then (2) has no eventually negative solutions.

Theorem 3 Consider the delay differential equation

$$
\begin{gathered}
{\left[x(t)-\sum_{j=1}^{n} c_{j} x\left(t-\theta_{j}\right)\right]^{\prime}+a(t) g\left[x\left(t-\sigma_{1}(t)\right), x\left(t-\sigma_{2}(t)\right), \ldots, x\left(t-\sigma_{k}(t)\right)\right]} \\
\quad+p(t) f\left[x\left(t-\tau_{1}(t)\right), x\left(t-\tau_{2}(t)\right), \ldots, x\left(t-\tau_{k}(t)\right)\right]=0,
\end{gathered}
$$

Where $a(t), p(t), \sigma_{i}(t) \tau_{i}(t) c_{j}, \theta_{j}, f$ and $g$ satisfy the condition of Theorem $1, i=1,2, \ldots, k . j=1,2, \ldots, n$. Then $(3)$ has only oscillatory solutions.

Proof From the result of Theorem 1 it follows that (3) has no eventually positive solutions. Also, from the result of Theorem 1 it follows that (3) has no eventually negative solutions. Therefore (3) has only oscillatory solutions.

\section{Applications}

Example 1 Consider the first order nonlinear functional differential equation 


$$
\left[x(t)-\frac{1}{3} x(t-1)\right]^{\prime}+x\left(t-\cos ^{2} t\right)+3[x(t-1)]^{1 / 3}\left[x(t-(\cos t+2)]^{2 / 3}=0\right.
$$

Where $\mathrm{c}=\frac{1}{3}, \theta=1, \mathrm{p}(\mathrm{t}) \equiv 3, \mathrm{a}(\mathrm{t}) \equiv 1, \mathrm{~m}=1$ and $\mathrm{M}=3$, Clearly, conditions of Theorem 3 are satisfied, then (13) has only oscillatory solutions.

Example 2 Consider the first order nonlinear functional differential equation

$$
\left[x(t)-\frac{1}{2} x\left(t-\frac{1}{3}\right)\right]^{\prime}+x\left(t-\sin ^{2} t\right)+2[x(t-1)]^{2 / 5}\left[x(t-(2 \cos t+3)]^{3 / 5}=0\right.
$$

where $\mathrm{c}=\frac{1}{2}, \theta=\frac{1}{3}, \mathrm{p}(\mathrm{t}) \equiv 2, \mathrm{a}(\mathrm{t}) \equiv 1, \mathrm{~m}=1$ and $\mathrm{M}=5$, Clearly, conditions of Theorem 3 are satisfied, Then (14) has only oscillatory solutions.

\section{Conclusion}

In this paper, we study the oscillatory behavior of solutions of a kind of first-order neutral nonlinear functional differential equations with time delay arguments. We look for the sufficient conditions of the existence of no eventually positive solutions and no eventually negative solutions for the delay functional differential inequalities. We obtain some result by the auxiliary function method which is important tools in oscillation theory. We also get some corollaries about the linear case: $\mathrm{f}(\mathrm{t}, \mathrm{x}(\mathrm{t}-\sigma))=\mathrm{x}(\mathrm{t}-\sigma)$.

\section{References}

[1] G. Ladas and I. P. Stavroulakis, On Delay Differential Inequalities of First Order, Funkcialaj Ekvacioj, 25 (1982), 105-113.

[2] L. C. Lin and G. Q. Wang, A Class Of Nonlinear Differential Inequalities With Time Delay Arguments, Annals of Differential Equations, Vol. 7 No. 1 (1991), 58-67.

[3] Weng Peixuan, Oscillations Of Solutions Of First-order Neutral Differential Difference Equations, Annals of Differential Equations, Vol. 7 No. 1 (1991), 103-116.

[4] Ruan Jiang, Oscillations of Neutral Differential Difference Equations With Several Retarded Arguments, Scientia Sinica (Series A) 5 (1986), 467-477.

[5] Busenberg S, Cooke K L. Models of vertically transmitted diseases with sequential continuous dynamics [A]. In: Lakshmikantham V, ed. Nonlinear Phenomena in Mathematical Sciences [M]. New York: Academic Press, 1982. 179-187.

[6] Tomaras A. Oscillatory behavior of an equation arising from an industrial problem Bull. Austral. Math. Soc. 13 (1975), 255-260.

[7] G. Ladas, Sharp conditions for oscillation caused by delays, Applicable Analysis, 9 (1979), 93-98.

[8] K. L. Cooke and J. Wiener, Retarded differential equations with piecewise constant delays, J. Math. Anal. Appl. 99 (1984), 265-297.
[9] A. R. Aftabizadeh and J. Wiener, oscillatory properties of first order linear functional differential equation, Applicable Anal. 20 (1985), 165-187.

[10] G. S. Ladde and B. G. Zhang, oscillation and non-oscillation for systems of two first order linear differential equations with delay, J. Math. Anal. Appl. 115 (1986), 57-75.

[11] K. L. Cooke and J. Wiener, An equation alternately of retarded and advanced type, Proc. Amer. Math. Soc. 99 (1987), 726-732.

[12] J. Wiener and A. R. Aftabizadeh, Differential equations alternately of retarded and advanced type, J. Math. Anal. Appl. 129 (1988), 243-255.

[13] Joseph Wiener, oscillations in systems of differential equations with piecewise constant argument, J. Math. Anal. Appl. 137 (1989), 221-239.

[14] B. G. Zhang and N. Pathi. oscillatory and non-oscillatory properties of first order differential equations with piecewise constant deviating arguments, J. Math. Anal. Appl. 139 (1989), 23-25.

[15] Y. Kitamure and T. Kusano, Oscillation of first order nonlinear differential equations with deviating argument, Proc. Amer. Math. Soc. 78 (1990), 64-68.

[16] K. Gopalsamy, M. R. S. Kulenovic and G. Ladas, On a logistic equation with piecewise constant arguments, Differential and Integral equations, 4 (1991), 215-223.

[17] Y. G. Sficas and I. P. Starvoulakis, Necessary and sufficient conditions of neutral differential equations, J. Math. Anal. Appl. 123 (1987), 494-507.

[18] B. G. Zhang, oscillation of first order neutral functional differential equations, J. Math. Anal. Appl. 2 (1989), 311-318.

[19] R. Yuan, Almost period solutions of neutral differential equations with piecewise constant delays, Chinese Ann. Math, 1998, 19A (4):499-506.

[20] Muminov, I. M., On the method of finding periodic solutions of second-order neutral differential equations with piecewise constant arguments, Adv. Differ. Equ. (2017) 2017:336.

[21] Shyam S. S., Tripathy, A. K., On oscillatory first nonlinear neutral differential equations with nonlinear impulses, J. Appl. Math. Comput. 2018.

[22] Tripathy, A. K., Shyam S. S., Pulsatile constant and characterization of first order neutral impulsive differential equations, Commun. Appl. Anal. 20 (2016), 65-76. 
[23] Candan, T.: Existence of non-oscillatory solutions to first-order neutral differential equations. Elect. J. Diff. Equ. 2016 (39), pp1-11.

[24] Candan, T.: Existence of positive solutions higher-order nonlinear neutral equations. J. Ineq. Appl. 2013, 573 (2013).

[25] G. Infante, R. Koplatadze, and I. P. Stavroulakis, Oscillation criteria for differential equations with several retarded arguments, Funkcial Ekvac., 58, No. 3, pp347-364 (2015).

[26] E. Braverman, G. E. Chatzarakis, and I. P. Stavroulakis, Iterative oscillation tests for differential equations with several non-monotone arguments, Adv. Differ. Equ. 2016, 87, pp1-18. (2016).

[27] K. Chudinov, Note on oscillation conditions for first-order delay differential equations, Electron. J. Qual. Theory Differ. Eqs. 2, pp1-10 (2016).
[28] Diblik, J., Positive solutions of nonlinear delayed differential equations with impulses, Appl. Math. Lett. 72, pp16-22 (2017).

[29] Tripathy, A. K., Santra S. S., Pinelas, S, Necessary and sufficient conditions of for asymptotic behavior of solutions of a class of first order impulsive systems, Adv. Dyn. Syst. Appl. 11 (2), pp135-145 (2016).

[30] Tripathy, A. K., Santra S. S., Necessary and sufficient conditions of for oscillations of solutions of a class of first order impulsive differential equations, Funct. Differ. Equ. 22 (3-4), pp149-167 (2015).

[31] K. M. Chudinov, Exact conditions of oscillation of solutions to differential equations with several Delays, J. Math. Sci. Vol. 230, pp790-793 (2018).

[32] A. Ozbekler, A. Zafer, Wong's oscillation theorem for the second-order delay differential equations, J. Math. Sci. Vol. 222, pp304-311 (2017). 